OPEN ACCESS

Edited by:

Xiangru Zhu,

Henan University, China

Reviewed by:

Qiwei Yang,

Chengdu Medical College, China

Wenbo Luo,

Liaoning Normal University, China

*Correspondence:

Dan Li

Lidantina@163.com

Jinping Liu

ljp202518@163.com

tThese authors have contributed equally to this work and share first

authorship

Specialty section:

This article was submitted to Personality and Social Psychology, a section of the journal

Frontiers in Psychology

Received: 08 June 2020

Accepted: 06 May 2021

Published: 03 June 2021

Citation:

Yin H, Cui X, Bai Y, Cao G

Zhang L, Ou Y, Li D and Liu J (2021)

The Effects of Angry Expressions and Fearful Expressions on Duration

Perception: An ERP Study.

Front. Psychol. 12:570497.

doi: 10.3389/fpsyg.2021.570497

\section{The Effects of Angry Expressions and Fearful Expressions on Duration Perception: An ERP Study}

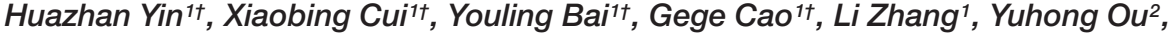

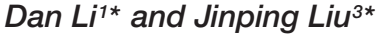 \\ 1 Cognition and Human Behavior Key Laboratory of Hunan Province, Hunan Normal University, Changsha, China, ${ }^{2}$ School \\ of Education Science, Nantong University, Nantong, China, ${ }^{3}$ Hunan Provincial Key Laboratory of Intelligent Computing \\ and Language Information Processing, Hunan Normal University, Changsha, China
}

Little is known about the electrophysiological basis of the effect of threat-related emotional stimuli with different motivational direction on duration perception. Thus, event-related potentials were employed to examine the effects of angry expressions and fearful expressions on perception of different duration (490-910 ms). Behavioral results showed there was a greater underestimation of the duration of angry expressions (approach-motivated negative stimuli) than fearful expressions (withdrawal-motivated negative stimuli), compared with neutral expressions. Event-related potentials results showed that, the area of Contingent Negative Variation (CNV) evoked by angry expression, fearful expression and neutral expression gradually increased. These results indicated that specific electrophysiological mechanisms may underlie the attention effects of angry and fearful expressions on timing. Specifically, compared with neutral expressions, fearful expressions and angry expressions both are likely to distract more attentional resources from timer, in particular, angry expressions attract more attention resources than fearful expressions from timer. The major contribution of the current study is to provide electrophysiological evidences of fear vs. anger divergence in the aspect of time perception and to demonstrate beyond the behavioral level that the categorization of threat-related emotions should be refined so to highlight the adaptability of the human defense system.

Keywords: threat-related, time perception, anger, fear, event-related potential

\section{INTRODUCTION}

Timing is a basic ability of human beings that enables us to adapt to the objective environment (Wittmann, 2009). When facing threat-related negative stimuli, human beings would change subjective timing to adapt to the objective environment (Droit-Volet and Gil, 2009). For example, when individual encounters threat stimuli (e.g., snakes, spiders, etc.), perceived duration is extended so that the individual has enough time to take the next action. At present, although researchers have known about the effect of threat-related negative emotional stimulus on the perception of time, however, little is known about the effect of different subtypes of threat-related negative stimuli on duration processing (Lake et al., 2016). Thus, the present study intends to continue to explore this question. It not only lays the foundation for revealing the influence 
of threat-related emotion on temporal perception, but also helps people to make better use of emotional timing rules to adapt to the environment.

The pacemaker-accumulator (PA) model, as one of the classical theoretical models explaining timing process (Gibbon et al., 1984), postulates the existence of an internal pacemaker that emits pulses with a certain frequency to an accumulator. There may be a switch manipulated by attention resources controlling the transmission of pulses (Meck, 1984; LeDoux, 2012). More precisely, pulses are passed into the accumulator when one pays attention to timing, and pulses are blocked when one does not pay attention to timing. According to previous studies, there are three kinds of mechanism of threat-related emotional stimuli on duration perception (Schirmer, 2011). Firstly, increasing arousal leads to overestimation of duration. Secondly, emotional stimulation can induce attention orientation, and then lead to the change of latency of timing switch, which finally lead to overestimation of duration. Third, when the emotional stimulus is a timing signal, attention to emotional stimulus will lead to overestimation of duration, and when the emotional stimulus is a distractor, the duration will be shortened. In short, arousal and attention are two important sources of variation affecting timing (Gibbon, 1977; Gibbon et al., 1984).

It is more and more recognized that threat-related emotions should be examined separately because each discrete emotion may have a specific function and consequently a specific attention-related effect on duration perception (Frijda, 1988; Vaish et al., 2008; Droit-Volet and Gil, 2009; Krusemark and $\mathrm{Li}, 2011)$. However, direct comparison of duration perception between different threat-related emotions remains very few (Tipples, 2008). Fear refers to the bad feeling that put oneself in worry about impending danger and intensely urge to protect oneself to get out of that situation (Vaish et al., 2008), while anger represents a collection of certain kinds of stimuli that forecast dangers (such as an unfulfilled wish or frustrated behavior) in the environment, which could bring about physical and psychological harm to the individual (Lerner and Keltner, 2000). Previous research has proved that physiological and behavioral responses and cognitive processes induced by fearful and angry stimuli particularly were divergent: the pattern of heart rate increasing caused by anger and fear is similar, but the peripheral vascular function of anger and fear is different (Ekman et al., 1983; Levenson, 1992). Since fearful stimuli promote withdrawal behavior, and angry stimuli promote approach behavior (Hutcherson and Gross, 2011), and the theory motivational dimension of emotions hold that the intensity of the motivation of emotional stimulation plays a key role in the allocation of attention resources (Bradley et al., 2001). Thus, the present study was designed to examine the attention-related time distortions caused by these two subtypes of threat-related emotional stimuli. In consideration of arousal and attention are two important sources of variation affecting timing (Gibbon, 1977; Gibbon et al., 1984), and some studies found that the arousal of the emotional expression may also influence timing (Droit-Volet and Meck, 2007; Stetson et al., 2007; Grommet et al., 2011; Lambrechts et al., 2011). Therefore, the arousal of the threatrelated negative emotional stimuli in this study was carefully controlled so as to prevent our results from being contaminated by the arousal differences among different emotional stimuli conditions. Furthermore, since the contributions of attention and arousal are often difficult to disentangle at the behavioral level, the event-related potentials (ERPs) were used to search for the neural correlates of the different underlying processes about influencing on duration perception by angry and fearful stimuli. To this day, a few studies have explored the effect of emotion stimuli on temporal perception using ERP technology. For example, one study found that the average amplitude of contingent negative variation $(\mathrm{CNV})$ induced by emotional conditions (anger and happy) was lower than that induced by neutral condition. In addition, under the emotional conditions, the P160 and P240 amplitudes were enhanced and the N230 amplitude was decreased (Gan et al., 2009). These findings suggest that temporal processing can be modulated by emotion stimulus, even within $200 \mathrm{~ms}$ of the stimulus onset, and that the attentional bias for emotion stimulus attenuates the cognitive resources for time perception. Other study directly investigated the less frequently considered role of attention as an alternative mediator of these effects with ERPs. In this study participants were asked to produce short intervals (0.9, $1.5,2.7$, and $3.3 \mathrm{~s}$ ) while viewing high arousal images with pleasant and unpleasant contents in comparison to neutral images. The valence-specificity of affective attention revealed by ERPs combined with behavioral timing results suggested that attention processes indeed contribute to emotion-induced temporal distortions, especially for longer target intervals (Tamm et al., 2014). Morever, there's another study also explored the different effects between two threat-associated emotions (fear and disgust) on duration perception (Zhang et al., 2014). These findings indicated that specific neural mechanisms may underlie the attention effects of different subtypes of threat-related emotions on timing, compared with neutral faces, fearful faces are likely to attract more attentional resources while disgusted faces may attract less attentional resources for emotional processing. In view of the dimension of emotional motivation, fearful expressions and angry expressions were different motivational direction (approaching or withdrawing) of emotional stimuli, which can infer that the attention-related mechanisms of time perception aroused by fear and anger are distinct, resulting in distinct ERP patterns when participants are asked to estimate the duration presented by fearful and angry expressions. The present study was to record the ERPs changes in the course of temporal generalization task and emotional stimuli with arousal controlled was employed to verify how fear and anger affect duration perception.

According to previous literature (Macar and Vidal, 2004; Zhang et al., 2014), we analyzed three ERP components: vertex positive potential (VPP), N170, and CNV. VPP is a large positive ERP with a latency of $170 \mathrm{~ms}$ and elicited at fronto-central electrodes (Jeffreys, 1996), and N170 is a large negative ERP with a latency of $170 \mathrm{~ms}$ and elicited at lateral posterior electrodes (Bentin et al., 1996). The main feature of these two scalp components is their enhanced response to faces compared to other multiple object categories (e.g., Bentin et al., 1996; Jeffreys, 1996; Rossion et al., 2003). Many studies have also proposed 
that N170, coordinated with emotions, created less amplitudes elicited in reaction to neutral facial expressions than emotional facial expressions, which correspond to its positive counterpart component of VPP (Batty and Taylor, 2003; Blau et al., 2007; Schyns et al., 2007). More importantly, numerous studies have also demonstrated that there was a positive correlation between the average amplitude of $\mathrm{CNV}$ and length of the estimated duration (Macar and Vidal, 2003) and the area of CNV was a positive correlation with the attentional resources of timer in different emotional sitmulus conditions (Zhang et al., 2014). Therefore, It is expected that early ERP components such as N170 and VPP would be enhanced by emotions, and that the area amplitudes of the slow CNV component may display separated waveforms in fear and anger conditions, indicating specific neural mechanisms underlying the attention effects of different subtypes of threat-related emotions on duration perception.

\section{METHODS}

\section{Participants}

Through G-power3.1 software (alpha level 0.05, power 0.8, effect size 0.3, subject group 1) (Faul et al., 2007), the total number of participants was calculated as 24 . Thus, a total of 30 undergraduate students (16 female, 14 male; mean age $=21.9$ years, range $=18-23)$ taken part in the experiment are from Southwest Normal University, Chongqing, China. All participants had normal or corrected-to-normal vision and had no prior neurological/psychiatric history. The study received written consent from every participant who was paid for their participation. This study was reviewed and approved by the local ethics committee of Southwest Normal University.

\section{Stimuli}

The experiment included a learning stage and a testing stage. A white oval was used as the stimulus of "target" duration for the learning phase. During the testing phase, 60 black-andwhite photos (20 angry, 20 fearful, and 20 neutral expressions) consisting of the same number of facial images for males and females from the native Chinese Facial Affective Picture System (CFAPS) were used as the stimulus of "probe" duration and presented at the center of computer monitoring (Gong et al., 2011). The valence and arousal score of each expression was asked to evaluate on a nine-point scale, and analyzed to see if the two types of negative expressions were significantly different from the neutral expressions. The main effect of the expression type was present $[F(2,38)=45.468, p<0.001$, $\eta^{2} p=0.705$; mean \pm standard deviation: angry $=3.10 \pm 0.50$, fearful $=2.95 \pm 0.45$, neutral $=4.21 \pm 0.53$; angry/fearful vs neutral: $p s<0.001]$ in valence, while no significant difference among the arousal scores of the three types of expressions $\left[F(2,38)=0.116, p=0.841, \eta^{2} p=0.006\right.$; angry $=5.18 \pm 1.32$, fearful $=5.30 \pm 0.82$, neutral $=5.17 \pm 0.44$; angry vs. neutral: $p=0.964$; fearful vs. neutral: $p=0.527$ ] occurred. Note that the chosen 20 neutral expressions have a generally higher arousal scores in comparison with the rest of the neutral expressions in the CFAPS to prohibit our results from being polluted by difference in arousal among emotional states [there was a sum of 422 neutral expressions in the CFAPS (valence $=4.29 \pm 0.52$; arousal $=3.84 \pm 0.69)]$.

\section{Procedure}

After visiting the laboratory, each participant was seated about $70 \mathrm{~cm}$ in front of a DELL computer in a special experimental room. The computer (19-inch CRT monitor, refresh rate $100 \mathrm{~Hz}$ ) was responsible for the presentation of the experimental stimuli and recording the participants' response data with E-Prime 2.0 (Psychology Software Tools, Inc., Pittsburgh, PA). Each stimulus was the same as each other in contrast and brightness $\left(4.0^{\circ} \times 4.6^{\circ}\right.$ visual angle $)$ against a black background. A temporal generalization task was employed to explore the timing mechanism (Pouthas et al., 2000; Macar and Vidal, 2004; Gil and Droit-Volet, 2011a,b). The task included a learning stage and a testing stage (Figure 1). We asked the participants to match a probe duration (equivalent to or not equivalent to) with a previously presented target duration. During the learning stage, participants were required to view a white oval that was the same size as the emotional images for the target duration (700 ms) 10 times on end. During the testing phase, participants were exposed to angry, fearful, or neutral pictures for the probe duration (490, 595, 700, 805, and $910 \mathrm{~ms}$ ) (Pouthas et al., 2000). In each trial, the participant had to press the " $D$ " or " $K$ " button (yes or no) on the computer keyboard to judge whether the probe duration was "the same" as the target duration or not. The testing stage was comprised of 12 blocks. Each block included 100 trials. Each probe duration $(490,595,700,805$, and $910 \mathrm{~ms})$ included 20 trials in each block. Between blocks is the balance between subjects, and the trails within blocks are random. In order to eliminate the interference between emotional stimuli, each block only contained one kind of trials to induce one emotion. Morever, to prohibit the participants from forgetting the target duration, it was presented six times in succession before the beginning of each block. The break between blocks was decided by selftermination of participants. In each trial, participants whose response latency was less than $100 \mathrm{~ms}$ or more than $1,500 \mathrm{~ms}$ were considered unavailable.

\section{EEG Recording and Analysis}

Recordings were made with a sampling frequency of $500 \mathrm{~Hz}$, an amplifier bandpass of $0.05-80 \mathrm{~Hz}$ ( $6 \mathrm{~dB} /$ octave), and an electrode impedance $5 \mathrm{k} \Omega$. Eye movements were subtracted (Semlitsch et al., 1986). Tin electrodes, mounted with the reference electrode on the left and right mastoids, were used to record electrical brain activity from 64 scalp sites in an elastic cap (Brain Products $\mathrm{GmbH}$, Gilching, Germany). Trials with EOG artifacts (mean EOG voltage exceeding $\pm 150 \mu \mathrm{V}$ ) and those contaminated with artifacts were excluded from averaging because of amplifier clipping, bursts of electromyographic activity, or peak-topeak deflection exceeding $\pm 150 \mu \mathrm{V}$. The data analysis and result revealed in this study were designed in Matlab R2014a (MathWorks, Natick, United States). In the present study, we primarily analyzed ERP produced by three facial expressions (anger, fear, and neutral) under five probe duration conditions and epochs changed after the onset of facial expressions within 


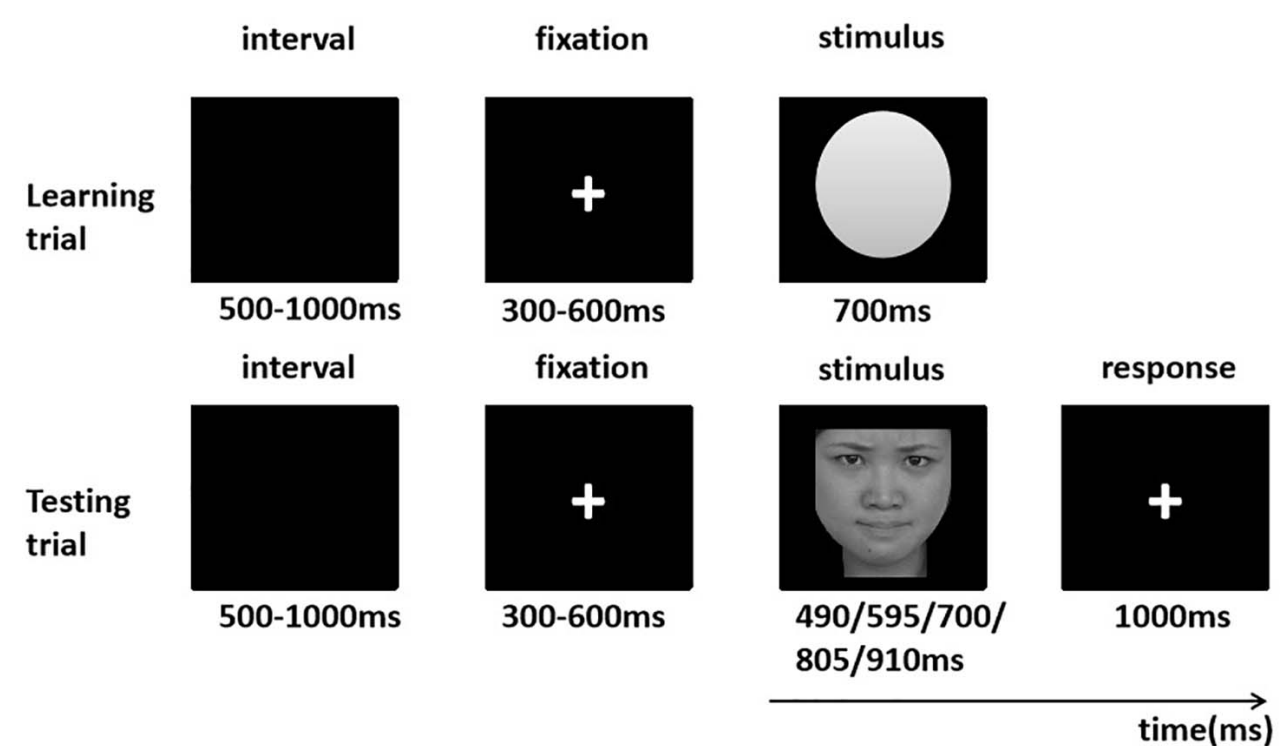

FIGURE 1 | Procedure of events in a sample learning and testing trial. The face picture was selected from the native Chinese Facial Affective Picture System (CFAPS) (Gong et al., 2011).

$1,200 \mathrm{~ms}$ from the baseline pre-stimulus at $200 \mathrm{~ms}$. On the basis of the relevant literature (Monfort et al., 2000; Pouthas et al., 2000), the mean amplitudes of fronto-central VPP, occipito-temporal $\mathrm{N} 170$, and the area amplitudes of the CNV-like component across different sets of electrodes were analyzed. Moreover, the electrode sites and time windows for each effect were chosen in line with grand-mean ERP topographies. About the VPP, three sites of FC1, FCz, and FC2 (time window $=170-190 \mathrm{~ms}$ ) were figured. For the N170, four sites of the mean amplitudes of P7, P8, PO7, and PO8 (time window $=170-190 \mathrm{~ms}$ ) were counted. For the CNV, three sites of the mean area amplitudes of FC1, FCz, and FC2 were counted. The CNV area amplitude is based on the integral part of the ERP waveform below the two zero intersections of the time axis (Macar and Vidal, 2003; Zhang et al., 2014).

\section{Statistics}

SPSS software 20.0 (IBM, Somers, United States) was used for statistical analysis. Mean \pm standard deviation are represented as descriptive data. A repeated measures ANOVA with a post hoc analysis was performed on behavioral data with affective category (angry, fearful, and neutral expressions) and probe duration $(490,595,700,805$, and $910 \mathrm{~ms})$ as factors. The significance level was set at 0.05. Where appropriate, the Greenhouse-Geisser correction was applied to ANOVA tests. Post hoc testing of significant main effects was conducted using Bonferroni method. A simple effects model was employed to explain the significant interactions. Partial eta-squared $\left(\eta^{2} p\right)$ was recorded to prove the effect size in ANOVA tests, with 0.0099 showing a small effect, 0.0588 displaying a medium effect, and 0.1379 indexing a large effect (Richardson, 2011). For assessing the Behavioral and ERP preformance of temporal generalization task, a Percentage of "yes" responses and response time, three affect-sensitive components were identified in stimulus-locked ERPs: VPP, N170, and CNV were calculated (Macar and Vidal, 2003).

\section{RESULTS}

\section{Behaviors}

\section{Percentage of "Yes" Responses}

A 3 (discrete emotion: anger, fear, and neutral) $\times 5$ (duration: 490, 595, 700, 805, and $910 \mathrm{~ms}$ ) repeated-measures ANOVA was used to analyze the proportion of "yes" responses (Figure 2). The interaction effect of discrete emotion by probe duration was highly present $\left[F(8,232)=34.240 ; p<0.001 ; \eta_{p}^{2}=0.541\right]$, meaning that the temporal distortion induced by two threatrelated emotional stimuli were dependent on the probe duration. Simple effect analysis showed that the proportion of "same" responses was modulated by emotion in $490-[F(2,58)=5.341$; $\left.p=0.007 ; \eta_{p}^{2}=0.156\right], 595-[F(2,58)=30.119 ; p<0.001$; $\left.\eta_{p}^{2}=0.509\right], 700-\left[F(2,58)=84.282 ; p<0.001 ; \eta^{2}{ }_{p}=0.744\right], 805-$ $\left[F(2,58)=30.368 ; p<0.001 ; \eta^{2} p=0.512\right], 910-[F(2,58)=6.800$; $\left.p=0.002 ; \eta^{2}{ }_{p}=0.190\right] \mathrm{ms}$. In particular, $46.2 \%$ angry, $50.6 \%$ fearful, and 56.0\% neutral expressions under $595 \mathrm{~ms}$ condition were estimated as having a duration of $700 \mathrm{~ms}$, while $60.8 \%$ angry, 58.7\% fearful, and 53.3\% neutral expressions under $805 \mathrm{~ms}$ condition were estimated as having a duration of $700 \mathrm{~ms}$. The pattern proportion of "yes" responses showed that, compared with neutral expression, the duration of fearful expression and angry expression were estimated to be shorter. Morever, a main effect of probe duration was significant $[F(4,116)=110.939$; $p<0.001 ; \eta_{p}^{2}=0.793$ ], indicating that the proportion of "yes" responses was smaller in the $490 \mathrm{~ms}(M=0.403 \pm 0.021)$ and $910 \mathrm{~ms}$ conditions $(M=0.461 \pm 0.023)$, compared to the $595 \mathrm{~ms}$ 


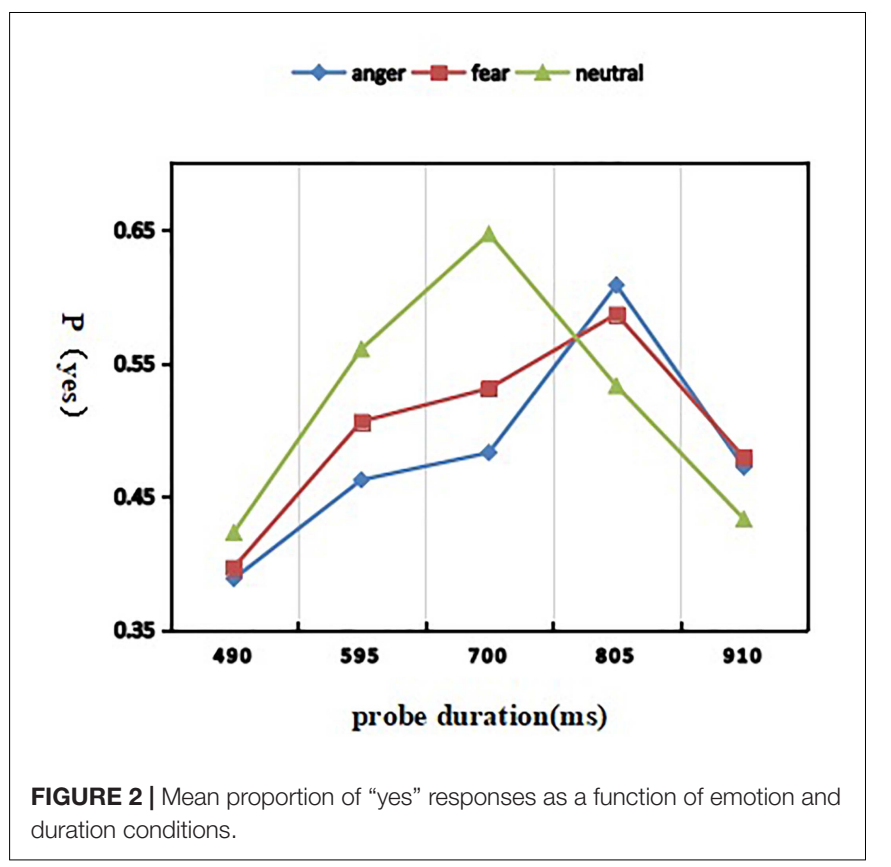

$(M=0.510 \pm 0.026), 700 \mathrm{~ms}(M=0.553 \pm 0.032)$, and $805 \mathrm{~ms}$ conditions $(M=0.576 \pm 0.034)(p s<0.001)$. The other main effect of emotional expression was present $[F(2,58)=25.680$; $\left.p<0.001 ; \eta^{2} p=0.470\right]$. Post hoc analyses revealed that, compared with the duration of neutral expression $(M=0.519 \pm 0.025)$, the duration of angry expression $(M=0.483 \pm 0.025)$ was more underestimation than that of fear expression $(M=0.500 \pm 0.023)$.

\section{Response Time (RT)}

A 3 (discrete emotion: anger, fear, vs. neutral) $\times 5$ (duration: 490, 595, 700, 805, vs. $910 \mathrm{~ms}$ ) repeated-measures ANOVA was used to analyze the response time (Figure 3 ). There was a significant main effect of probe duration $[F(4,116)=146.630 ; p<0.001$; $\left.\left.\eta_{p}^{2}=0.835\right)\right]$. Post hoc analyses revealed that, the RT went down with probe duration $\left(\mathrm{M}_{490}=507 \pm 46 \mathrm{~ms}, \mathrm{M} 595=469 \pm 41 \mathrm{~ms}\right.$, $\mathrm{M}_{700}=448 \pm 38 \mathrm{~ms}, \mathrm{M}_{805}=404 \pm 32 \mathrm{~ms}, \mathrm{M}_{910}=373 \pm 30 \mathrm{~ms}$ in $490,595,700,805$, and $910 \mathrm{~ms}$ conditions) going on. Both the effect of discrete emotion $[F(2,58)=0.167 ; p=0.846]$ and the interaction effect of discrete emotion by probe duration failed to reach significance $[F(8,232)=0.195 ; p=0.991]$.

\section{Stimulus-Locked Affective ERP Components}

Table 1 indicates descriptive statistical results of affective effects in all three stimulus-locked components (VPP, N170, and CNV).

\section{$V P P$}

A 3 (discrete emotion: anger, fear, vs. neutral) $\times 5$ (duration: 490, 595, 700, 805, vs. $910 \mathrm{~ms}) \times 2$ (hemisphere: left, right) repeated-measures ANOVA was employed to analyze the average amplitudes of the VPP component. There was a significant main effect of discrete emotion was present, $[F(2,58)=442.244$, $\left.p<0.001, \eta_{p}^{2}=0.938\right]$, indicating that the VPP amplitude induced by neutral expressions $(M=4.223 \pm 0.035 \mu \mathrm{V})$, fearful expressions $(M=4.570 \pm 0.039 \mu \mathrm{V})$, and angry expressions $(M=5.035 \pm 0.044 \mu \mathrm{V})$ gradually increased significantly

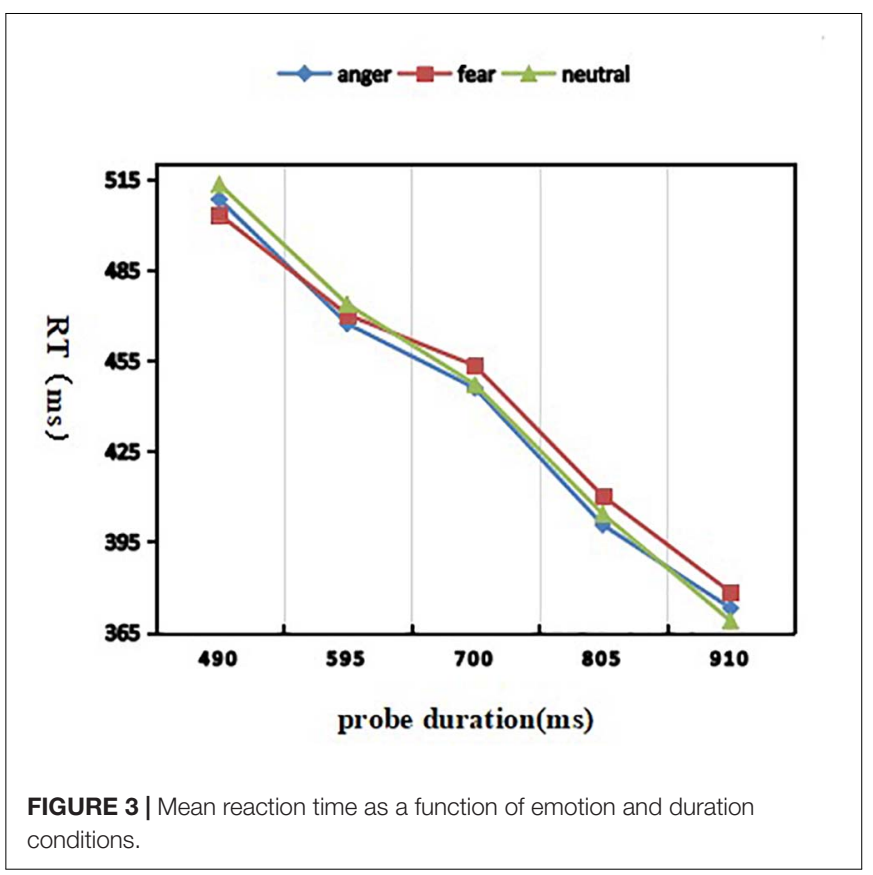

TABLE 1 | Event-related potentials VPP, N170, and CNV for different experimental stimuli across the nrobe durations.

\begin{tabular}{|c|c|c|c|c|}
\hline \multicolumn{2}{|c|}{ Probe duration (ms) } & \multicolumn{3}{|c|}{ Angry expression Fearful expression } \\
\hline \multirow{5}{*}{ VPP } & $490 \mathrm{~ms}$ & $5.032(0.043)^{\star * \#}$ & $4.542(0.038)^{\star \star}$ & $4.204(0.032)$ \\
\hline & $595 \mathrm{~ms}$ & $5.121(0.050)^{\star * \#}$ & $4.562(0.021)^{\star \star}$ & $4.267(0.036)$ \\
\hline & $700 \mathrm{~ms}$ & $4.984(0.052)^{\star * \#}$ & $4.613(0.032)^{\star \star}$ & $4.229(0.049)$ \\
\hline & $805 \mathrm{~ms}$ & $5.038(0.048)^{\star \star \#}$ & $4.564(0.037)^{\star \star}$ & $4.174(0.039)$ \\
\hline & $910 \mathrm{~ms}$ & $4.999(0.056)^{\star \star \#}$ & $4.571(0.042)^{\star \star}$ & $4.242(0.036)$ \\
\hline \multirow{5}{*}{ N170 } & $490 \mathrm{~ms}$ & $-4.088(0.043)^{* * \#}$ & $-3.409(0.038)^{\star \star}$ & $-3.146(0.032)$ \\
\hline & $595 \mathrm{~ms}$ & $-3.999(0.050)^{k \star \#}$ & $-3.388(0.021)^{\star \star}$ & $-3.083(0.036)$ \\
\hline & $700 \mathrm{~ms}$ & $-4.136(0.052)^{k \star \#}$ & $-3.337(0.032)^{\star \star}$ & $-3.121(0.049)$ \\
\hline & $805 \mathrm{~ms}$ & $-4.082(0.048)^{k \star \#}$ & $-3.386(0.037)^{\star \star}$ & $-3.176(0.039)$ \\
\hline & $910 \mathrm{~ms}$ & $-4.121(0.056)^{k \star \#}$ & $-3.379(0.042)^{\star \star}$ & $-3.108(0.036)$ \\
\hline \multirow{5}{*}{ CNV } & $490 \mathrm{~ms}$ & $1.879(0.035)^{\star * \#}$ & $2.082(0.048)^{\star \star}$ & $2.249(0.031)$ \\
\hline & $595 \mathrm{~ms}$ & $2.101(0.063)^{\star * \#}$ & $2.405(0.059)^{\star \star}$ & $2.737(0.050)$ \\
\hline & $700 \mathrm{~ms}$ & $2.258(0.052)^{* * \#}$ & $2.55(0.045)^{\star \star}$ & $2.986(0.036)$ \\
\hline & 805 ms & $2.397(0.063)^{\star \star \#}$ & $3.070(0.069)^{\star \star}$ & $3.476(0.046)$ \\
\hline & $910 \mathrm{~ms}$ & $2.544(0.044)^{* * \#}$ & $3.063(0.062)^{\star \star}$ & $3.581(0.079)$ \\
\hline
\end{tabular}

${ }^{* *} p<0.001 ;{ }^{*} p<0.01$; significant difference from the Neutral stimuli; ${ }^{*} p<0.001$; significant difference from the fearful stimuli. VPP $(\mu \mathrm{V}) ; N 170(\mu \mathrm{V})$; and CNV $(\mu \mathrm{V} \cdot \mathrm{S})$; mean values with standard deviations.

( $p s<0.001)$. Morever, the main effect of the hemisphere also was significant $\left[F(1,29)=63.158, p<0.001, \eta^{2}{ }_{p}=0.685\right]$, indicating that the average amplitudes of the VPP in the left hemisphere $(M=4.577 \pm 0.040 \mu \mathrm{V})$ were significantly smaller than the right hemisphere $(M=4.642 \pm 0.041 \mu \mathrm{V})$. However, the other effects were not significant ( $p>0.05$, Figure 4).

\section{N170}

A 3 (discrete emotion: anger, fear, vs. neutral) $\times 5$ (duration: 490, $595,700,805$, vs. $910 \mathrm{~ms}) \times 2$ (hemisphere: left, right) repeatedmeasures ANOVA was used to analyze the average amplitudes of N170 component. There was a significant main effect of 


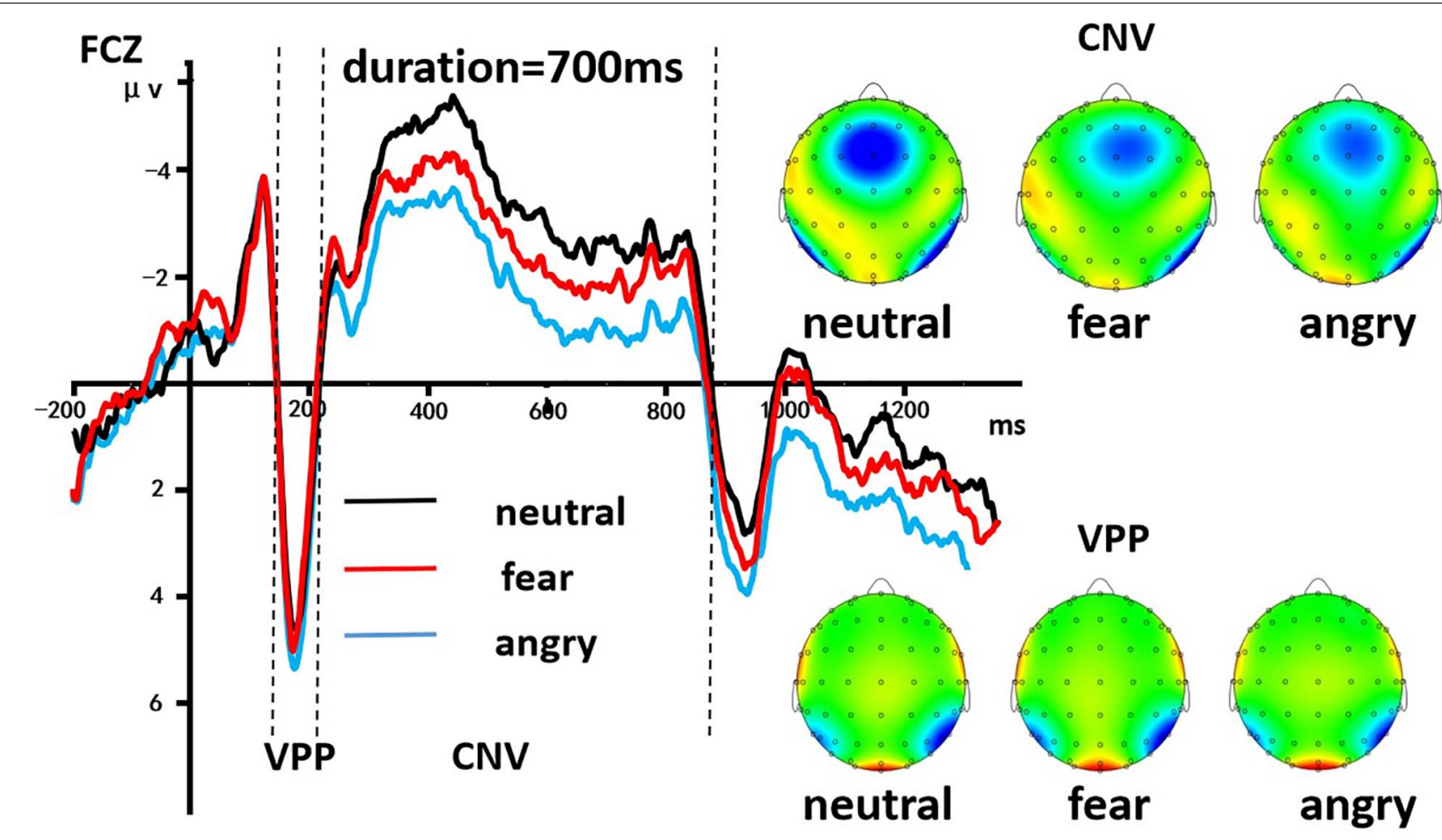

FIGURE 4 | The grand-mean ERP waveforms of the VPP and the CNV components. The CNV area amplitude was based on the integral part of the ERP waveform below the two zero intersections of the time axis. Mean amplitudes of the VPP component were calculated during 170-190 ms. The black line indicated neutral emotion, the red line indicated fear emotion, and the blue line indicated angry emotion.

discrete emotion was present, $[F(2,58)=588.714, p<0.001$, $\left.\eta_{p}^{2}=0.953\right]$, indicating that the N170 amplitude induced by neutral expressions $(M=-3.690 \pm 0.032 \mu \mathrm{V})$, fearful expressions $(M=-3.942 \pm 0.036 \mu \mathrm{V})$, and angry expressions $(M=-$ $4.645 \pm 0.042 \mu \mathrm{V})$ gradually increased significantly $(p s<0.001)$. Morever, The main effect of the hemisphere was significant $[F(1$, $\left.29)=11,691.782, p<0.001, \eta_{p}^{2}=0.998\right]$, indicating that the average amplitudes of the N170 in the left hemisphere $(M=-$ $3.568 \pm 0.030 \mu \mathrm{V})$ were significantly smaller than the right hemisphere $(M=-4.617 \pm 0.043 \mu \mathrm{V})$. However, the other effect were not significant $(p>0.05$, Figure 5).

\section{CNV}

A 3 (discrete emotion: anger, fear, vs. neutral) $\times 5$ (duration: 490, 595, 700, 805, vs. $910 \mathrm{~ms}) \times 2$ (hemisphere: left, right) repeated-measures ANOVA was used to analyze the average amplitudes of the $\mathrm{CNV}$ area. There was a significant main effect of discrete emotion, $[F(2,58)=198.651, p<0.001$, $\left.\eta_{p}^{2}=0.873\right]$, indicating the CNV area that induced by neutral expressions $(M=3.006 \pm 0.028 \mu \mathrm{V} \cdot \mathrm{s})$, fearful expressions $(M=2.634 \pm 0.025 \mu \mathrm{V} \cdot \mathrm{s})$, and angry expressions $(M=2.236 \pm 0.022 \mu \mathrm{V} \cdot \mathrm{s})$ gradually decreased significantly $(p s<0.001)$. The main effect of the hemisphere was also significant $\left[F(1,29)=23,684.294, p<0.001, \eta^{2} p=0.999\right]$, indicating that the average amplitudes of the CNV area in the left hemisphere $(M=2.190 \mu \mathrm{V} \cdot \mathrm{s})$ were significantly smaller than the right hemisphere $(M=3.060 \pm 0.029 \mu$
$\mathrm{V} \cdot \mathrm{s})$. In addition, The main effect of probe duration was also significant, $\left[F(4,116)=325.321, p<0.001, \eta_{p}^{2}=0.918\right]$, with post hoc analyses indicating that the CNV area for $490 \mathrm{~ms}(M=2.070 \pm 0.019 \mu \mathrm{V} \cdot \mathrm{s})$ was smaller than for $595 \mathrm{~ms}(M=2.414 \pm 0.023 \mu \mathrm{V} \cdot \mathrm{s})$; the $\mathrm{CNV}$ area for $595 \mathrm{~ms}$ was smaller than for $700 \mathrm{~ms}(M=2.598 \pm 0.026 \mu \mathrm{V} \cdot \mathrm{s})$. However, there was no difference between the area amplitude of $\mathrm{CNV}$ at 700, $805(M=2.981 \pm 0.028 \mu \mathrm{V} \cdot \mathrm{s})$, and $910 \mathrm{~ms}$ $(M=3.062 \pm 0.031 \mu \mathrm{V} \cdot \mathrm{s})$. The interaction effect of discrete emotion $\times$ duration was significant $[F(8,232)=7.457, p<0.001$, $\left.\eta^{2} p=0.205\right]$. Simple effect analysis indicated that the effect of emotion on the CNV area first increased, then decreased with the increasing duration of stimulus presentation $[F(2$, $\left.58)=19.235 ; p<0.001 ; \eta_{p}^{2}=0.399\right]$ in 490-ms condition; $\left[F(2,58)=25.514 ; p<0.001 ; \eta^{2} p=0.468\right]$ in 595-ms condition; $\left[F(2,58)=58.789 ; p<0.001 ; \eta^{2} p=0.670\right]$ in $700-m s$ condition; $\left[F(2,58)=71.132 ; p<0.001 ; \eta_{p}^{2}=0.710\right]$ in $805 \mathrm{~ms}$ condition; $\left[F(2,58)=64.360 ; p<0.001 ; \eta^{2}{ }_{p}=0.689\right]$ in $910-\mathrm{ms}$ condition (Figure 4).

\section{DISCUSSION}

The main aim of the current study was to investigate the effects of two threat-related emotional stimulus, which are arousal-controlled angry expressions and fearful expressions on duration perception with ERPs. Behavioral results revealed that compared with neutral expression, fearful expression and 


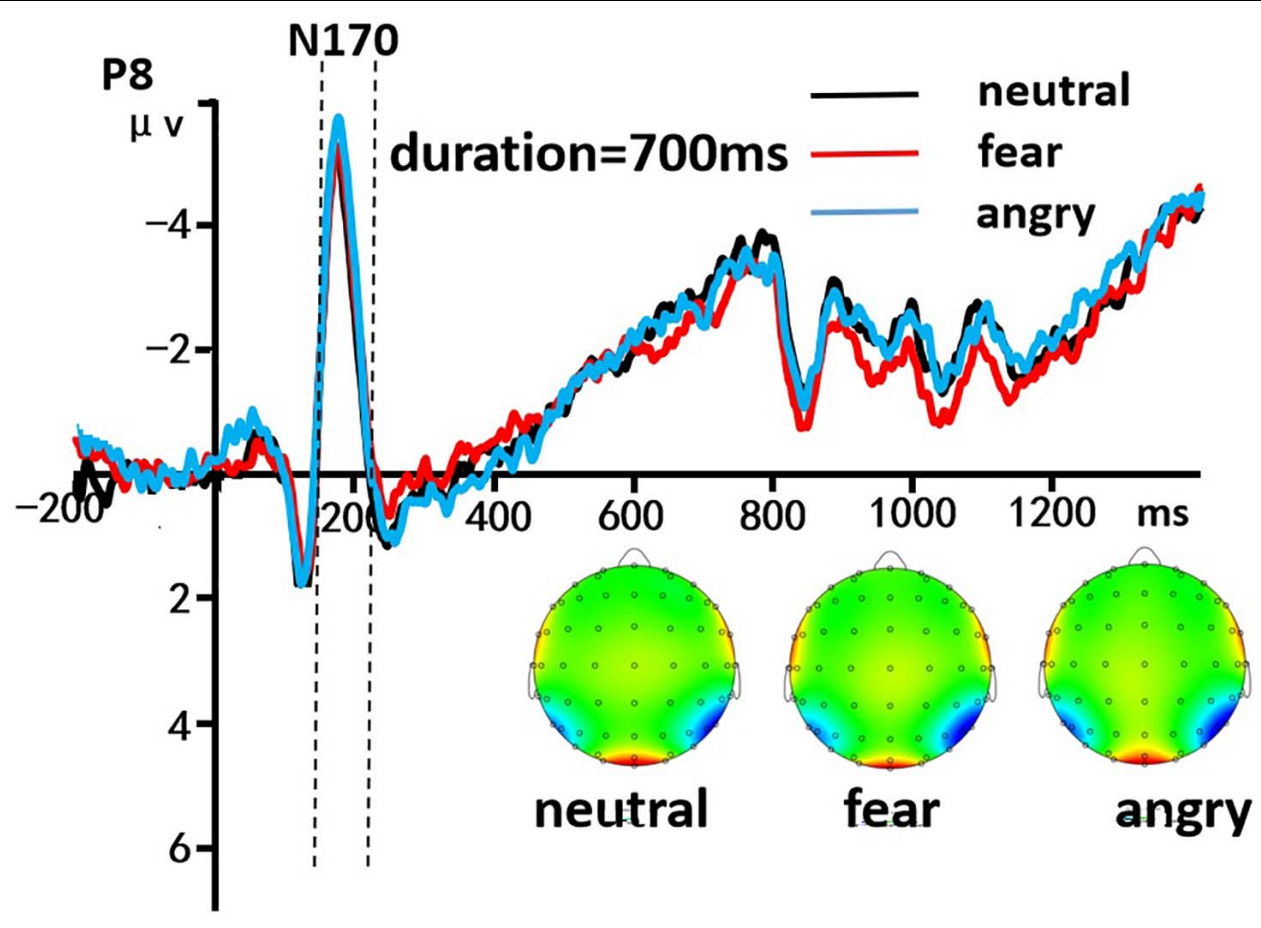

FIGURE 5 | The grand-mean ERP waveforms of the N170 component. Mean amplitudes of the N170 component were calculated during $170-190$ ms. The black line indicated neutral emotion, the red line indicated fear emotion, and the blue line indicated angry emotion.

angry expression were being estimated shorter. Several stimuluslocked ERP components were used to objectively characterize the states generated by affective expressions. Accordingly, the ERP data revealed that the early stage of emotion modulated duration perception was represented by larger frontocentral VPP amplitudes and occipito-temporal N170 amplitudes in fear/angry expression conditions, compared with neutral condition. Morever, the CNV, which is an online index of timing, displayed separated waveforms in different emotional stimuli conditions, with smaller amplitudes in angry and fearful conditions when compared with neutral condition.

The behavioral data in this study indicated that the underestimation of angry expression and fearful expression was remarkable, because previous behavioral studies often found that the duration of emotional expression was overestimated compared with neutral expression (Gil et al., 2007; Tipples, 2008; Gil and Droit-Volet, 2011a; Fayolle et al., 2015). According to the PA model, the distort of duration perception is often explained in terms of arousal-induced and attention-related mechanisms (Matell and Meck, 2000; Droit-Volet et al., 2004; Droit-Volet and Meck, 2007; Wittmann and Paulus, 2008). The mechanism of arousal-induced supposed that the internal clock was accelerated theoretically by the physiological arousal level of emotional stimuli, leading to a great number of accumulated pulses, thus bringing about an overestimation of duration perception (Droit-Volet et al., 2004; Noulhiane et al., 2007). After controlling the arousal ratings across the three emotional conditions, this study mainly investigated the attention-related time distortions caused by fearful and angry expression. It was hypothesized that, compared with neutral expression, fearful expression and angry expression attract more attention to the emotional content of the stimuli and thus divert processing resources from the timer, resulting in an underestimation of time. Consistent with this hypothesis, the behavioral data showed that the duration of fearful expression and angry expression were judged shorter than that of neutral expression. Similarly, there were another two behavioral studies controlled the arousal levels of negative pictures (mainly fearful ones, e.g., spiders or rats) when investigating the effect of emotion on timing, which found that negative pictures were overestimated in higharousal condition (arousal range $=6.5-7.5$ ) while they were underestimated in low-arousal condition (arousal range $=4$ 5.7), compared with low-arousal neutral pictures (Angrilli et al., 1997). This opposite effect of negative pictures as a function of arousal suggested that the attention-related mechanism mainly works for low arousing stimuli whereas the arousal-induced mechanism mainly works for high arousing stimuli (Angrilli et al., 1997; Buhusi and Meck, 2006; Droit-Volet and Meck, 2007). In this study, the arousal of emotional expression is just a low arousal condition (arousal scores in this study: angry expressions $=5.18 \pm 1.32$; fearful expressions $=5.30 \pm 0.82$ ) Thus, we investigated in this study per se relatively low arousing stimuli of fear and anger so as to explore the attention-related mechanism of duration perception. In addition, there might be another explanation of motivational direction influencing the perception of duration (Gable et al., 2017). Anger arises in situations where approach toward a goal is interrupted or an anticipated reward is blocked (Carver and Scheier, 2008), and 
that approach motivation in negative affective states related to the perception of time shortening. However, the underestimation of the duration of fear expression was not consistent with previous studies (e.g., Gable et al., 2017). The study found that a withdrawal-motivated negative state (disgust) caused the perception of time to lengthen relative to a neutral state, and fear belongs to a type of withdrawal-motivated negative state. Therefore, the effect of motivational direction of emotional stimulation on duration perception is still a problem that needs to be further explored.

In addition, a particularly noteworthy finding was that the duration estimation of angry expression was shorter than that of fear expression. It has been discussed in the literature that fearful and angry expressions could evoke different physiological responses and cognitive processes. Anger evokes similar patterns with injections of epinephrine and nor-epinephrine combined, and the patterns evoked by fear and injections of epinephrine are similar. The interconnection of anger to physiological variables was significantly higher than in the case of fear, showing that it is integrated more during anger (AX, 1953). This may mean that angry expression stimuli attract more attention resources from the time dimension of emotional stimuli than fearful expression stimuli, which leads to more underestimation.

The ERP data in this study found that larger fronto-central VPP amplitudes in fearful/angry expression conditions than in the neutral condition were represented in the early stage of emotion-modulated time perception. Many studies have compared emotional and neutral expressions which has alike arousal but higher ratings in emotional expressions (Batty and Taylor, 2003; Williams, 2006; Blau et al., 2007), albeit VPP was proves to be regulated by emotional expressions for smaller amplitudes in neutral expressions than in emotional expressions (such as fear) (Williams, 2006; Schyns et al., 2007). A natural covariation entre arousal and the valence grades of emotional stimuli occurred in the manipulation (i.e., a U-shaped connection in valence-arousal coordinate). In these studies, the effects of emotional-controlled found on ERP components could be caused by diversification of values or changes in arousal, or both. Accordingly, this study also discovered that the amplitudes of VPP could be raised by the valence of facial expressions separately. Fortunately, the occipito-temporal N170 amplitudes are also modulated by emotional expressions. In line with the early structural encoding operations described by Bruce and Young (1986), early ERP components (e.g., $\mathrm{N} 170$ ) are thought to reflect rapid structural encoding of faces. This may mean that the structure of emotional expressions is more complex than that of neutral expressions. Furthermore, the most novel ERP finding of this study is that the main effect of emotion was significant, suggesting that the CNV area in the neutral condition $(M=3.006 \mu \mathrm{V} \cdot \mathrm{s})$ was greater than that in the fear condition $(M=2.634 \mu \mathrm{V} \cdot \mathrm{s})$ and angry condition $(M=2.236 \mu \mathrm{V} \cdot \mathrm{s})$. The medial fronto-central cortex (typically at the electrode site of $\mathrm{FCz}$ ) before the supplementary motor area (SMA) was considered to be the neurosubstrate of the timing function reflected in the CNV (Macar and Vidal, 2004). The SMA showed consistent activation in temporal processing (Coull et al., 2008). Furthermore, the vitality of the
SMA was heightened by diverting attention to timing optionally (Coull et al., 2008). On the basis of such thesis, it can be known to all that the degree of attention turned to timing determined the level of neural activation that contributes to time perception (Macar and Vidal, 2004). As a result, compared with neutral expressions, a smaller CNV area was observed in the fearful condition and was more apparent in the angry condition. This indicated that emotional processing occupied more attentional resources, resulting in underestimation of time. Therefore, compared with CNV results, despite both fearful and angry expressions being threat-related, different attention deviation effects were observed in the two emotional conditions. The current study contributed different neural correlates between angry expressions and fearful expressions on duration perception, and demonstrates that it cannot focus on behavioral aspects but refine the classification of threat-associated emotions to stress the fitness of the human defense system (Krusemark and Li, 2011).

Finally, a non-linearity was displayed in the interaction effect of emotion by stimulus duration on the CNV area: with the growing duration of stimuli, the effect of emotion increased at first but then decreased. Thus, the effect of emotion on the perception of time appears to be greater in the medium durations $(595,700$, and $805 \mathrm{~ms})$ than in the excessive durations (490 and $910 \mathrm{~ms}$ ). This CNV pattern which showed the duration from 300 to $800 \mathrm{~ms}$ was the highest sensitivity on time perception is congruent with previous findings. The main effect of stimulus duration was also significant. The post hoc analyses indicated that the CNV area for $490 \mathrm{~ms}$ was smaller than for $595 \mathrm{~ms}$, which is smaller than for $700 \mathrm{~ms}$; however, there is no difference between the area amplitude of CNV at 805 and $910 \mathrm{~ms}$. This result is consistent with that of previous research (Pfeuty et al., 2005). The CNV results implied that the attentional mechanism could not interpret the pattern of all data. It is necessary to conduct further researches to inspect more factors that influence temporal perception. In particular, one of the major shortcomings of this study is that it did not monitor the subjects' basic conditions such as depression, anxiety and mood, which may pollute the results of this study.

\section{CONCLUSION}

In summary, arousal-controlled emotional stimuli was used in the current study to verify and compare the effect of two subtypes of threat-related emotional expression on duration perception. We suggested that angry and fearful expressions had different effects on the attention-associated mechanism of time perception, which is issued in divergent behavioral and ERP patterns. Particularly, there was a greater underestimation of the timing of angry expressions than fearful expressions when compared with neutral expressions. ERP results showed that, compared with neutral expressions, a smaller CNV area was observed in the fearful expression condition and angry expression condition, and the trend was more apparent in the angry expression condition. We propose that it is necessary to refine the categorization of threat-related emotions in order to emphasize the adaptability of 
human defense systems to optimize actions in response to various environmental hazards.

\section{DATA AVAILABILITY STATEMENT}

The raw data supporting the conclusions of this article will be made available by the authors, without undue reservation.

\section{ETHICS STATEMENT}

The studies involving human participants were reviewed and approved by the Ethics Committee Southwest Normal University. The patients/participants provided their written informed consent to participate in this study.

\section{REFERENCES}

Angrilli, A., Cherubini, P., Pavese, A., and Mantredini, S. (1997). The influence of affective factors on time perception. Percept. Psychophys. 59, 972-982. doi: 10.3758/bf03205512

AX, A. F. (1953). The physiological differentiation between fear and anger in humans. Psychosomatic Med. 15, 433-442. doi: 10.1097/00006842-19530900000007

Batty, M., and Taylor, M. J. (2003). Early processing of the six basic facial emotional expressions. Cogn. Brain Res. 17, 613-620. doi: 10.1016/s0926-6410(03)001745

Bentin, S., Allison, T., Puce, A., Perez, A., and McCarthy, G. (1996). Electrophysiological studies of face perception in humans. J. Cogn. Neurosci. 8, 551-565. doi: 10.1162/jocn.1996.8.6.551

Blau, V. C., Maurer, U., Tottenham, N., and McCandliss, B. D. (2007). The facespecific N170 component is modulated by emotional facial expression. Behav. Brain Funct. 3, 1-13.

Bradley, M. M., Codispoti, M., Cuthbert, B. N., and Lang, P. J. (2001). Emotion and motivation : defensive and appetitive reactions in picture processing. Emotion 1, 276-298. doi: 10.1037/1528-3542.1.3.276

Bruce, V., and Young, A. (1986). Understanding face recognition. Br. J. Psychol. 77, 305-327. doi: 10.1111/j.2044-8295.1986.tb02199.x

Buhusi, C. V., and Meck, W. H. (2006). Interval timing with gaps and distracters: evaluation of the ambiguity, switch, and time-sharing hypotheses. J. Exp. Psychol. Animal Behav. Proc. 32, 329-344. doi: 10.1037/0097-7403.32.3.329

Carver, C. S., and Scheier, M. F. (2008). "Feedback processes in the simultaneous regulation of action and affect," in Handbook of Motivation Science, eds J. Y. Shah and W. L. Gardner (New York, NY: Guilford Press), 308-324.

Coull, J. T., Nazarian, B., and Vidal, F. (2008). Timing, storage, and comparison of stimulus duration engage discrete anatomical components of a perceptual timing network. J. Cogn. Neurosci. 20, 2185-2197. doi: 10.1162/jocn.2008. 20153

Droit-Volet, S., Brunot, S., and Niedenthal, P. (2004). Perception of the duration of emotional events. Cogn. Emot. 18, 849-858. doi: 10.1080/02699930341000194

Droit-Volet, S., and Gil, S. (2009). The time-emotion paradox. Philos. Trans. Royal Soc. Biol. Sci. 364, 1943-1953.

Droit-Volet, S., and Meck, W. H. (2007). How emotions colour our perception of time. Trends Cogn. Sci. 11, 504-513. doi: 10.1016/j.tics.2007.09.008

Ekman, P., Levenson, R. W., and Friesen, W. V. (1983). Autonomic nervous system activity distinguishes among emotions. Science 221, 1208-1210. doi: 10.1126/ science.6612338

Faul, F., Erdfelder, E., Lang, A. G., and Buchner, A. (2007). G*Power 3: a flexible statistical power analysis program for the social, behavioral, and biomedical sciences. Behav. Res. Methods 39, 175-191. doi: 10.3758/bf03193146

Fayolle, S., Gil, S., and Droit-Volet, S. (2015). Fear and time: fear speeds up the internal clock. Behav. Proc. 120, 135-140. doi: 10.1016/j.beproc.2015.09.014

Frijda, N. H. (1988). The laws of emotion. Am. Psychol. 43, 349-356.

\section{AUTHOR CONTRIBUTIONS}

$\mathrm{HY}, \mathrm{YB}, \mathrm{DL}, \mathrm{XC}$, and JL designed and coordinated the study. YB, GC, LZ, XC, and YO carried out experiments and data process. GC drafted the manuscript. HY reviewed the manuscript. All authors gave the final approval for publication.

\section{FUNDING}

This work was supported by the National Natural Science Foundation of China under Grant Nos. 31671125 and 61971188, in part by the Natural Science Fund of Hunan Province under Grant No. 2018JJ3349.

Gable, P. A., Neal, L. B., and Poole, B. D. (2017). Sadness speeds and disgust drags: influence of motivational direction on time perception in negative affect. Motivat. Sci. 2, 238-255. doi: 10.1037/mot0000044

Gan, T., Wang, N., Zhang, Z., Li, H., and Luo, Y. J. (2009). Emotional influences on time perception: evidence from event-related potentials. Neuroreport 20, 839-843. doi: 10.1097/wnr.0b013e32832be7dc

Gibbon, J. (1977). Scalar expectancy theory and Weber's law in animal timing. Psychol. Rev. 84, 279-325. doi: 10.1037/0033-295x.84.3.279

Gibbon, J., Church, R. M., and Meck, W. H. (1984). Scalar timing in memory. Annals N. Y. Acad. Sci. 423, 52-77. doi: 10.1111/j.1749-6632.1984.tb23417.x

Gil, S., and Droit-Volet, S. (2011a). Time flies in the presence of angry expressions. depending on the temporal task used! Acta Psychol. (Amst) 136, 354-362. doi: 10.1016/j.actpsy.2010.12.010

Gil, S., and Droit-Volet, S. (2011b). Time perception in response to ashamed expressions in children and adults. Scand. J. Psychol. 52, 138-145. doi: 10.1111/ j.1467-9450.2010.00858.x

Gil, S., Niedenthal, P. M., and Droit-Volet, S. (2007). Anger and time perception in children. Emotion 7, 219-225. doi: 10.1037/1528-3542.7.1.219

Gong, X., Huang, Y. X., Wang, Y., and Luo, Y. J. (2011). Revision of the Chinese facial affective picture system. Commun. Mental Health J. 25, 40-46.

Grommet, E. K., Droit-Volet, S., Gil, S., Hemmes, N. S., Baker, A. H., and Brown, B. L. (2011). Time estimation of fear cues in human observers. Behav. Process 86, 88-93. doi: 10.1016/j.beproc.2010.10.003

Hutcherson, C. A., and Gross, J. J. (2011). The moral emotions: a social functionalist account of anger, disgust, and contempt. J. Personal. Soc. Psychol. 100, 719-725. doi: 10.1037/a0022408

Jeffreys, D. A. (1996). Evoked studies of face and object processing. Vis. Cogn. 3, 1-38. doi: 10.1080/713756729

Krusemark, E. A., and Li, W. (2011). Do all threats work the same way? divergent effects of fear and disgust on sensory perception and attention. J. Neurosci. 31, 3429-3434. doi: 10.1523/jneurosci.4394-10.2011

Lake, J. I., Labar, K. S., and Meck, W. H. (2016). Emotional modulation of interval timing and time perception. Neurosci. Biobehav. Rev. 64, 403-420. doi: 10.1016/ j.neubiorev.2016.03.003

Lambrechts, A., Mella, N., Pouthas, V., and Noulhiane, M. (2011). Subjectivity of time perception: a visual emotional orchestration. Front. Int. Neurosci. 5:73. doi: 10.3389/fnint.2011.00073

LeDoux, J. E. (2012). Rethinking the emotional brain. Neuron 73, 653-676. doi: 10.1016/j.neuron.2012.02.004

Lerner, J. S., and Keltner, D. (2000). Beyond valence: toward a model of emotion specifific influences on judgement and choice. Cogn. Emot. 14, 473-493. doi: 10.1080/026999300402763

Levenson, R. W. (1992). Autonomic nervous system differences among emotions. Psychol. Sci. 3, 23-27. doi: 10.1111/j.1467-9280.1992.tb00251.x

Macar, F., and Vidal, F. (2003). The CNV peak: an index of decision making and temporal memory. Psychophysiology 40, 950-954. doi: 10.1111/1469-8986. 00113 
Macar, F., and Vidal, F. (2004). Event-related potentials as indices of time processing: a review. J. Psychophysiol. 18, 89-104. doi: 10.1027/0269-8803.18. 23.89

Matell, M. S., and Meck, W. H. (2000). Neuropsychological mechanisms of interval timing behavior. Bioessays 22, 94-103. doi: 10.1002/(sici)1521-1878(200001)22: $1<94$ ::aid-bies $14>3$.0.co;2-e

Meck, W. H. (1984). Attentional bias between modalities: effect on the internal clock, memory, and decision stages used in animal time discrimination. Annals N. Y. Acad. Sci. 423, 528-541. doi: 10.1111/j.1749-6632.1984.tb23457.x

Monfort, V., Pouthas, V., and Ragot, R. (2000). Role of frontal cortex in memory for duration: an event-related potential study in humans. Neurosci. Lett. 286, 91-94. doi: 10.1016/s0304-3940(00)01097-1

Noulhiane, M., Mella, N., Samson, S., Ragot, R., and Pouthas, V. (2007). How emotional auditory stimuli modulate time perception. Emotion 7, 697-704. doi: 10.1037/1528-3542.7.4.697

Pfeuty, M., Ragot, R., and Pouthas, V. (2005). Relationship between CNV and timing of an upcoming event. Neurosci. Lett. 382, 106-111. doi: 10.1016/j. neulet.2005.02.067

Pouthas, V., Garnero, L., Ferrandez, A. M., and Renault, B. (2000). ERPs and PET analysis of time perception: spatial and temporal brain mapping during visual discrimination tasks. Hum. Brain Mapp. 10, 49-60. doi: 10.1002/(sici)10970193(200006)10:2<49::aid-hbm10>3.0.co;2-8

Richardson, J. T. E. (2011). Eta squared and partial eta squared as measures of effect size in educational research. Educ. Res. Rev. 6, 135-147.

Rossion, B., Joyce, C. A., Cottrell, G. W., and Tarr, M. J. (2003). Early lateralization and orientation tuning for face, word, and object processing in the visual cortex. Neuroimage 20, 1609-1624. doi: 10.1016/j.neuroimage.2003.07.010

Schirmer, A. (2011). How emotions change time. Front. Int. Neurosci. 5:58. doi: 10.3389/fnint.2011.00058

Schyns, P. G., Petro, L. S., and Smith, M. L. (2007). Dynamics of visual information integration in the brain for categorizing facial expressions. Curr. Biol. 17, 1580-1585. doi: 10.1016/j.cub.2007.08.048

Semlitsch, H. V., Anderer, P., Schuster, P., and Presslich, O. (1986). A solution for reliable and valid reduction of ocular artifacts, applied to the P300 ERP. Psychophysiology 23, 695-703. doi: 10.1111/j.1469-8986.1986.tb00696.x
Stetson, C., Fiesta, M. P., and Eagleman, D. M. (2007). Does time really slow down during a frightening event? PLoS One 2:e19295. doi: 10.1371/journal. pone.0001295

Tamm, M., Uusberg, A., Allik, J., and Kreegipuu, K. (2014). Emotional modulation of attention affects time perception: evidence from eventrelated potentials. Acta Psychol. 149, 148-156. doi: 10.1016/j.actpsy.2014. 02.008

Tipples, J. (2008). Negative emotionality influences the effects of emotion on time perception. Emotion 8:127. doi: 10.1037/1528-3542.8.1.127

Vaish, A., Grossmann, T., and Woodward, A. (2008). Not all emotions are created equal: the negativity bias in social-emotional development. Psychol. Bull. 134, 383-402. doi: 10.1037/0033-2909.134.3.383

Williams, L. M. (2006). An integrative neuroscience model of "significance" processing. J. Int. Neurosci. 5, 1-47. doi: 10.1142/s0219635206001082

Wittmann, M. (2009). The inner experience of time. Philos. Trans. R. Soc. Biol. Sci. 364, 1955-1967.

Wittmann, M., and Paulus, M. P. (2008). Decision making, impulsivity and time perception. Trends Cogn. Sci. 12, 7-12. doi: 10.1016/j.tics.2007. 10.004

Zhang, D., Liu, Y., Wang, X., Chen, Y., and Luo, Y. (2014). The duration of disgusted and fearful faces is judged longer and shorter than that of neutral faces: the attention-related time distortions as revealed by behavioral and electrophysiological measurements. Front. Behav. Neurosci. 8:293. doi: 10.3389/ fnbeh.2014.00293

Conflict of Interest: The authors declare that the research was conducted in the absence of any commercial or financial relationships that could be construed as a potential conflict of interest.

Copyright (c) 2021 Yin, Cui, Bai, Cao, Zhang, Ou, Li and Liu. This is an open-access article distributed under the terms of the Creative Commons Attribution License (CC BY). The use, distribution or reproduction in other forums is permitted, provided the original author(s) and the copyright owner(s) are credited and that the original publication in this journal is cited, in accordance with accepted academic practice. No use, distribution or reproduction is permitted which does not comply with these terms. 